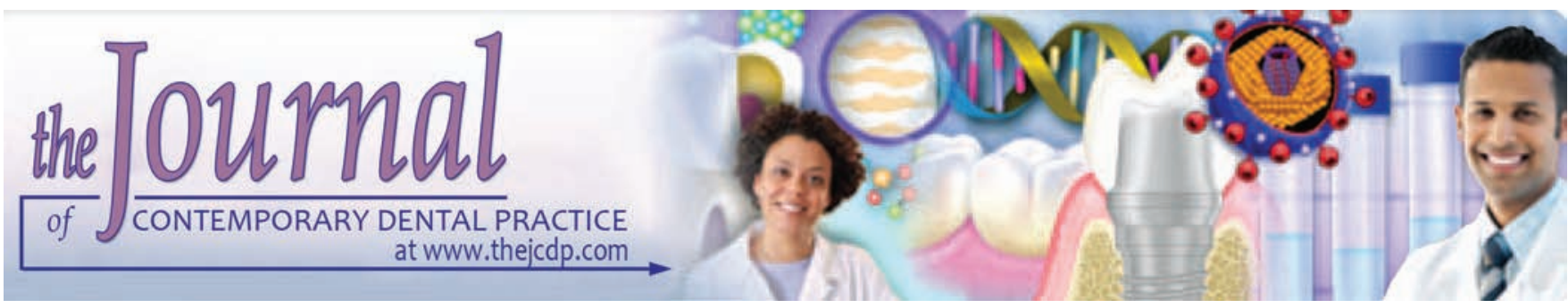

\title{
Force Decay Characteristics of commonly used Elastomeric Chains on Exposure to various Mouth Rinses with different Alcohol Concentration: An in vitro Study
}

${ }^{1} \mathrm{~S}$ Ramachandraiah, ${ }^{2} \mathrm{~K}$ Sridharan, ${ }^{3} \mathrm{~A}$ Nishad, ${ }^{4} \mathrm{KK}$ Manjusha, ${ }^{5}$ Esther A Abraham, ${ }^{6} \mathrm{M}$ Mohamed Ramees

\begin{abstract}
Aim: The aim of this study was to test the effect of varying alcohol concentrations of various commercially available mouthwashes on force decay of elastomeric chains in prestretched and unstretched condition.
\end{abstract}

Materials and methods: A total of five specimen groups were tested with a total sample size of 180 specimens. A specimen is described as a three-link, short module, clear elastomeric chain (3M Unitek, Ortho Plus, Ortho Organizer). The control and test groups were independently submerged in separate $37^{\circ} \mathrm{C}$ artificial saliva to simulate the oral conditions. Effects of different solution groups and time (initial, 1, 7, 14, 21, and 28 days) on three different elastomeric chains and force ( $\mathrm{cN}$ ) are analyzed with two-way analysis of variance; pair-wise comparisons are done by t-test.

Results: Higher mean force was recorded initially followed by 24 hours $>7$ days $>14$ days $>21$ days and 28 days respectively. The difference in mean force decay among the different time intervals was found to be statistically significant $(p<0.001)$. Among the different solutions, higher mean force was recorded in $21.6 \%$ alcohol mixture $>$ Listerine $8.38 \%>$ Wokadine $>$ alcohol mixture $8.38 \%>$ artificial saliva.

Conclusion: In conclusion, alcohol-containing mouth rinses cause an increase in force decay of elastomeric chain over time.

Clinical significance: These mouth rinses are commonly recommended during orthodontic treatment. The orthodontist can know better that the alcohol content of the mouth rinses and the structural and molecular modification results in the decay of elastomeric chain.

\footnotetext{
${ }^{1}$ Ram Dental Clinic, Tumkur, Karnataka, India

${ }^{2,5,6}$ Department of Orthodontics and Dentofacial Orthopedics Sri Siddhartha Dental College, Tumkur, Karnataka, India

${ }^{3,4}$ Department of Orthodontics and Dentofacial Orthopaedics Noorul Islam College of Dental Sciences, Thiruvananthapuram Kerala, India
}

Corresponding Author: S Ramachandraiah, Ram Dental Clinic, Tumkur, Karnataka, India, e-mail: drramdent@gmail.com
Keywords: Alcohol, Elastomeric chain, Force decay, Mouth rinses.

How to cite this article: Ramachandraiah S, Sridharan $\mathrm{K}$, Nishad A, Manjusha KK, Abraham EA, Ramees MM. Force Decay Characteristics of commonly used Elastomeric Chains on Exposure to various Mouth Rinses with different Alcohol Concentration: An in vitro Study. J Contemp Dent Pract 2017;18(9):812-820.

Source of Support: Nil

Conflict of Interest: None

\section{INTRODUCTION}

Synthetic elastomeric chains have been used by orthodontists since the 1960s. These polyurethane materials have largely replaced latex elastics, for intra-arch tooth movement and to consolidate spaces. ${ }^{1}$

Placement and removal of chain elastics require little chair time for the clinician and minimal patient cooperation during application. The synthetic elastomeric materials have also been found to be relatively compatible with the mucosa. These factors have contributed to high degree of professional acceptance of the chain elastics in orthodontics. $^{2}$

Elastomeric ligatures are manufactured in two basic forms: Injection molded and cut. The injection-molded ligature is made by injection of liquefied elastomeric material into a mold and curing, whereas the cut ligature is sliced from previously processed elastomeric tubing. ${ }^{3}$

The greatest degree of force loss occurred in the first 3 hours, with the remainder of force decay staying relatively constant over the next 21 days. In dry air, percentage of force decay after 21 days was illustrated to be 42 to $63 \%$ depending on the product brand. In addition, force decay occurred more quickly when elastomers were submerged in water bath. Another factor affecting the degree 
of force decay is the amount of initial load applied to the elastomers. As the initial load increased, the percentage of force decay also increased, the total remaining force at each time interval over 21 days was maintained at higher levels with increased initial loads. ${ }^{4}$

Mouth rinses should be used during the orthodontic mechanotherapy for antimicrobial activity, to decrease the plaque accumulation and thereby prevent demineralization. Chlorhexidine acts in a preventive manner in the reduction of bacterial plaque, in patients undergoing orthodontic treatment. It is a synthetic antimicrobial agent that presents a high level of activity, however, having secondary effects, such as staining and ulceration of mucosa. Hence, other mouth rinses, such as Listerine and Wokadine gargle are recommended during orthodontic treatment. The alcohol content of the mouth rinses has shown to cause structural and molecular modification, which results in the decay of elastomeric chain. ${ }^{4}$

However, the effect of ethanol at different concentrations as available in various mouthwashes on force degradation of elastomeric chains in prestretched and unstretched conditions needs further study.

Hence, the purpose of this study was to test the effect of varying alcohol concentrations of various commercially available mouthwashes on force decay of elastomeric chains in prestretched and unstretched condition.

\section{MATERIALS AND METHODS}

This study was carried out in the Department of Orthodontics and Dentofacial Orthopedics, Siddhartha Dental College, Tumkur, India. Sample for this study comprised 180 specimens. Alcohol-contained mouth rinses and elastomeric chain with short module, prestretched and unprestretched, were included in the study. Mouth rinses that are expired and physically damaged (broken) elastomeric chains were excluded from the study.

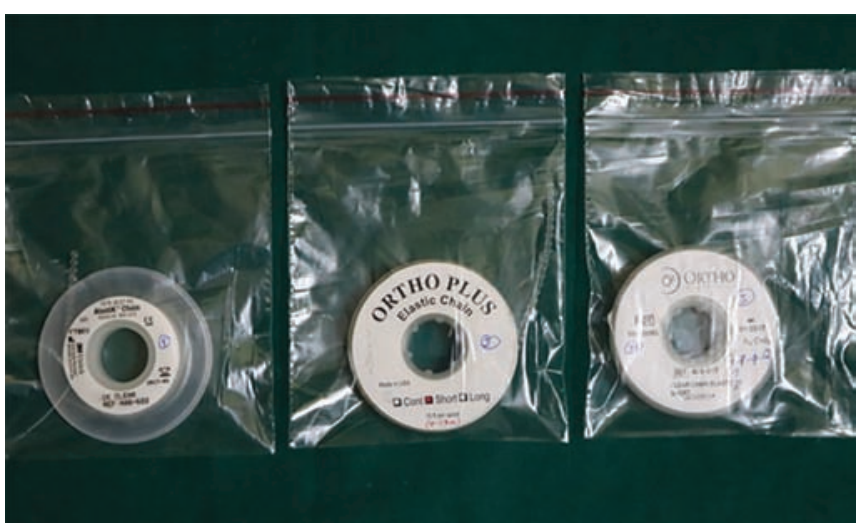

Fig. 1: Clear short elastomeric chains - 3M Unitek, Ortho Plus, Ortho Organizer

A total of five specimen groups were tested with a total sample size of 180 specimens. A specimen is described as a three-link, short module, clear elastomeric chain (3M Unitek, Ortho Plus, Ortho Organizer) (Fig. 1). Specimens were mounted on custom-made test jigs. Five custommade jigs, each with $18 \mathrm{~mm}$ apart for unprestretched and $22 \mathrm{~mm}$ apart for prestretched elastomeric chains were used to hold stretched elastomeric chains at a constant length.

The control and test groups were independently submerged in separate $37^{\circ} \mathrm{C}$ artificial saliva to simulate the oral conditions. The formulation of the saliva solution was made by Goran Pharma Pvt. Ltd. (Gujarat, India). It was housed in an incubator and maintained at room temperature using thermostat. Similarly, the test specimens were submerged in the respective test solutions, i.e., artificial saliva, Listerine, Wokadine, alcohol mixture $21.6 \%$, and alcohol mixture $8.38 \%$ (Figs $2 \mathrm{~A}$ and B) for 60 seconds, twice a day, for the entire 28-day test period.

Each 60-second exposure of the test specimens was measured using a digital clock and the two daily exposures were separated by 9 hours. After being submerged in the respective solutions, specimens were dipped in
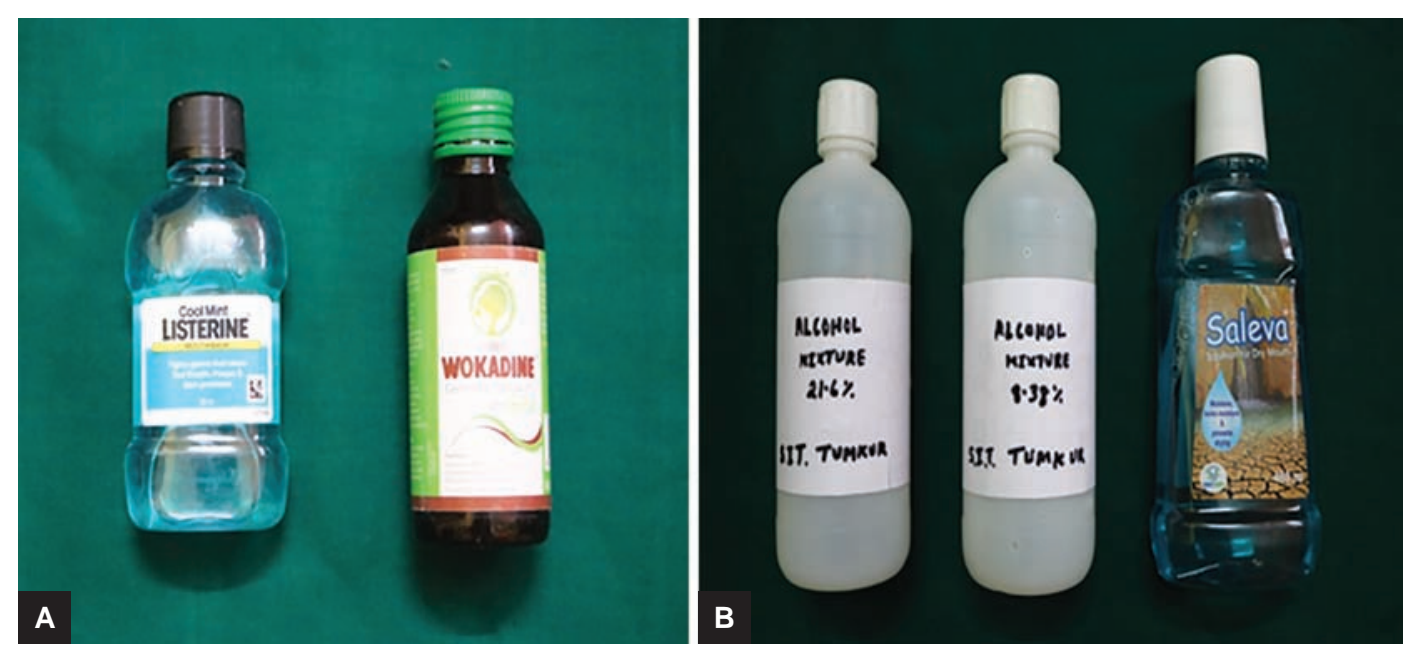

Figs 2A and B: Mouth rinses (test and control solutions) 


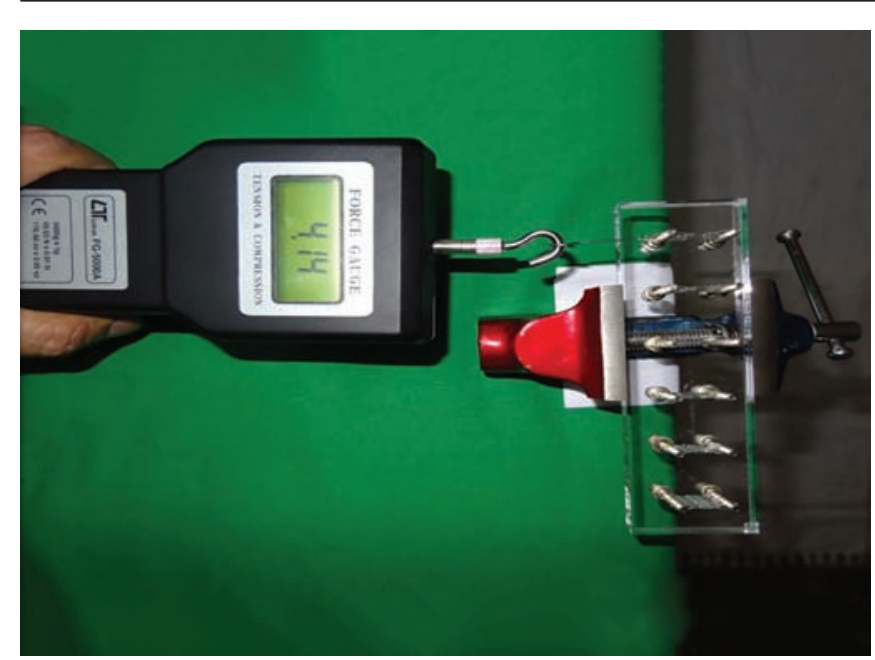

Fig. 3: Measurement of force

separate, distilled water baths for 10 seconds to simulate rinsing of the mouth rinses from the oral cavity. These specimens were then placed back into artificial saliva at $37^{\circ} \mathrm{C}$. The control group underwent the same protocol; however, these elastomeric chains were only exposed to artificial saliva.

Six test measurements of the remaining force were made at the following time intervals: Initial (0), 1, 7, 14, 21 , and 28 days. Force measurements were obtained with a digital force tester (Lutron FG-5000; Lutron Electronic Enterprise Co., Ltd., Taipei, Taiwan) (Fig. 3). Before the initiation of the study, the force tester was calibrated by measuring the weight of known items, to ensure the reliability of the instrument. After each measurement, the force tester was reset to a zero reading before taking the next measurement. During force measurement, the jigs were securely bound to a bench top using a vice clamp.

Measurements were made by leaving one end of the elastomeric chain secured on the pin and fixing the other to the force tester, allowing for the measurement of the tensile force. Measurement readings were taken with the elastomeric chain stretched to the same $18 \mathrm{~mm}$ apart in unprestretched and $22 \mathrm{~mm}$ apart in prestretched elastomeric chains length that the jig pins had previously maintained them. All chains were handled and measured in the same manner at the same vertical and horizontal distance on the jig board to ensure consistent measurements.

With five groups, each having six specimens at six time points, a total sample size of 180 specimens (36 specimens per group) were tested. Specimens were discarded after measurements were made. In the case that any elastomeric chains were to break during testing, an additional ten specimens were added to the control and test groups. Therefore, 230 specimens were used. The effects of group (artificial saliva, Listerine, Wokadine, alcohol mixture
$21.6 \%$, and alcohol mixture $8.38 \%$ ) and time (initial, 1 , $7,14,21$, and 28 days) on force were analyzed with a two-way analysis of variance (ANOVA).

\section{Statistical Analysis}

The statistical software namely SAS 9.2 and Statistical Package for the Social Sciences 15.0 was used for the analysis of the data. Statistical analysis was performed using descriptive statistics, such as mean, standard deviation, and standard error to describe the mean scores of the respondents. Effects of groups (artificial saliva, Wokadine, Listerine, alcohol concentration $21.6 \%$, and alcohol concentration 8.38\%) and time (initial, 1, 7, 14, 21 and 28 days) on three different elastomeric chains (3M Unitek, Ortho Plus, Ortho organizer) and force $(\mathrm{cN})$ are analyzed with two-way ANOVA; pair-wise comparisons are done by t-test.

\section{RESULTS}

As shown in Table 1 in Listerine, at 24 hours, highest force decay was recorded by prestretched Ortho Plus (57.75\%) and least by unstretched Ortho Organizer (40.58\%). At 28 days, among unstretched and prestretched elastomeric chains, unstretched has highest force decay than prestretched.

Table 2 reveals that in Wokadine, at 24 hours, highest force decay was recorded by unstretched Ortho Organizer (58.35\%) and least by prestretched 3M Unitek (39.92\%). At 28 days, among unstretched and prestretched elastomeric chains, unstretched has highest force decay than prestretched.

In alcohol mixture $21.6 \%$, at 24 hours, highest force decay was recorded by unstretched Ortho Organizer (55.18\%) and least by prestretched 3M Unitek (39.87\%). At 28 days, among unstretched and prestretched elastomeric chains, unstretched has highest force decay than prestretched (Table 3).

In Table 4 (alcohol mixture $8.38 \%$ ), at 24 hours, highest force decay was recorded by unstretched Ortho Plus (49.17\%) and least by prestretched 3M Unitek (39.87\%). At 28 days, among unstretched and prestretched elastomeric chains, unstretched has highest force decay than prestretched.

As shown in Table 5, in artificial saliva, at 24 hours, highest force decay was recorded by unstretched Ortho Plus (48.98\%) and least by prestretched 3M Unitek (39.45\%). At 28 days, among unstretched and prestretched elastomeric chains, unstretched has highest force decay than prestretched.

Table 6 reveals that among the durations, higher mean force was recorded initially followed by 24 hours $>7$ days $>14$ days $>21$ days and 28 days respectively. 
Table 1: Mean force decay ( $\mathrm{cN}$ ) recorded in Listerine during different time durations for all elastomeric chains

\begin{tabular}{|c|c|c|c|c|c|}
\hline Listerine & $\begin{array}{l}\text { Measurement time } \\
\text { points (days) }\end{array}$ & Mean $\pm S D$ & $\begin{array}{l}\text { Standard error } \\
\text { of mean }\end{array}$ & Minimum & Maximum \\
\hline \multirow[t]{5}{*}{ Unstretched 3M Unitek } & 1 & $273.31 \pm 3.66$ & 1.49 & 269.6 & 278.9 \\
\hline & 7 & $243.51 \pm 4.44$ & 1.81 & 239.6 & 249.7 \\
\hline & 14 & 219. $7 \pm 3.46$ & 1.41 & 215.3 & 224.4 \\
\hline & 21 & $206.18 \pm 7.19$ & 2.93 & 198.7 & 215.1 \\
\hline & 28 & $174.75 \pm 4.90$ & 2.00 & 168.7 & 180.8 \\
\hline \multirow[t]{5}{*}{ Prestretched 3M Unitek } & 1 & $211.5 \pm 2.33$ & 0.95 & 209.2 & 215.4 \\
\hline & 7 & $201.6 \pm 3.06$ & 1.25 & 198.3 & 205.6 \\
\hline & 14 & $176.91 \pm 1.59$ & 065 & 174.8 & 178.8 \\
\hline & 21 & $157.06 \pm 2.53$ & 1.03 & 152.2 & 158.9 \\
\hline & 28 & $143.96 \pm 3.13$ & 1.28 & 140.4 & 147.8 \\
\hline \multirow[t]{5}{*}{ Unstretched Ortho Plus } & 1 & $269.8 \pm 6.67$ & 2.72 & 258.3 & 276.4 \\
\hline & 7 & $236.83 \pm 3.40$ & 1.38 & 230.3 & 239.6 \\
\hline & 14 & $201.98 \pm 1.97$ & 0.87 & 200.2 & 205.0 \\
\hline & 21 & $189.8 \pm 1.32$ & 0.54 & 187.8 & 191.1 \\
\hline & 28 & $167.05 \pm 2.30$ & 0.94 & 164.2 & 170.0 \\
\hline \multirow[t]{5}{*}{ Prestretched Ortho Plus } & 1 & $210.95 \pm 2.46$ & 1.006 & 208.3 & 215.5 \\
\hline & 7 & $197.7 \pm 1.81$ & 0.74 & 193.5 & 198.7 \\
\hline & 14 & $173.85 \pm 1.54$ & 0.63 & 172.2 & 176.1 \\
\hline & 21 & $157.05 \pm 2.36$ & 0.96 & 152.9 & 159.8 \\
\hline & 28 & $147.18 \pm 3.20$ & 1.30 & 142.9 & 150.6 \\
\hline \multirow[t]{5}{*}{ Unstretched Ortho Organizer } & 1 & $263.94 \pm 5.58$ & 2.49 & 258.7 & 270.8 \\
\hline & 7 & $243.01 \pm 3.901$ & 1.59 & 239.2 & 249.3 \\
\hline & 14 & $216.25 \pm 4.30$ & 1.75 & 210.8 & 220.7 \\
\hline & 21 & $206.2 \pm 7.04$ & 2.87 & 198.8 & 215.3 \\
\hline & 28 & $174.7 \pm 4.76$ & 1.94 & 168.9 & 180.4 \\
\hline \multirow[t]{5}{*}{ Prestretched Ortho Organizer } & 1 & $209.7 \pm 5.05$ & 2.06 & 200.10 & 215.0 \\
\hline & 7 & $196.66 \pm 2.26$ & 0.92 & 192.7 & 198.6 \\
\hline & 14 & $175.78 \pm 3.50$ & 1.42 & 170.7 & 179.7 \\
\hline & 21 & $155.11 \pm 3.39$ & 1.38 & 150.7 & 159.6 \\
\hline & 28 & $145.9 \pm 3.16$ & 1.29 & 140.6 & 148.9 \\
\hline
\end{tabular}

SD: Standard deviation

Table 2: Mean force decay ( $\mathrm{cN}$ ) recorded in Wokadine during different time durations for all elastomeric chains

\begin{tabular}{llllll}
\hline Wokadine & Measurement time & & & & \\
points (day) & Mean \pm SD & Standard error & Minimum & Maximum \\
\hline Unstretched 3M Unitek & 1 & $294.2 \pm 3.71$ & 1.51 & 290.1 & 298.5 \\
& 7 & $263.36 \pm 4.42$ & 180 & 259.2 & 269.4 \\
& 14 & $244.9 \pm 7.43$ & 3.03 & 136.4 & 254.5 \\
Prestretched 3M Unitek & 21 & $228.58 \pm 8.12$ & 3.31 & 218.7 & 238.8 \\
& 1 & $195.05 \pm 5.08$ & 1.66 & 188.5 & 200.5 \\
& 7 & $224.8 \pm 2.45$ & 1.002 & 220.7 & 227.4 \\
Unstretched Ortho Plus & 14 & $201.6 \pm 3.06$ & 1.25 & 198.3 & 205.6 \\
& 21 & $191.58 \pm 2.61$ & 1.066 & 188.5 & 195.7 \\
& 1 & $165.46 \pm 4.46$ & 1.82 & 159.6 & 170.7 \\
& 7 & $141.0 \pm 5.81$ & 2.37 & 136.0 & 149.4 \\
& 14 & $289.7 \pm 5.79$ & 2.36 & 279.7 & 296.8 \\
& 21 & $157.43 \pm 1.42$ & 0.58 & 256.0 & 259.3 \\
& 28 & $225.6 \pm 4.07$ & 1.67 & 221.2 & 231.4 \\
& & $216.51 \pm 5.34$ & 2.18 & 210.1 & 225.5
\end{tabular}




\begin{tabular}{|c|c|c|c|c|c|}
\hline Wokadine & $\begin{array}{l}\text { Measurement time } \\
\text { points (day) }\end{array}$ & Mean $\pm S D$ & Standard error & Minimum & Maximum \\
\hline \multirow[t]{5}{*}{ Prestretched Ortho Plus } & 1 & $226.2 \pm 1.83$ & 0.74 & 223.4 & 228.6 \\
\hline & 7 & $201.55 \pm 3.93$ & 1.60 & 197.2 & 106.7 \\
\hline & 14 & $190.41 \pm 4.20$ & 1.71 & 185.4 & 195.6 \\
\hline & 21 & $162.76 \pm 3.82$ & 1.56 & 158.6 & 168.5 \\
\hline & 28 & $146.38 \pm 3.13$ & 1.27 & 140.4 & 149.2 \\
\hline \multirow[t]{5}{*}{ Unstretched Ortho Organizer } & 1 & $306.8 \pm 3.86$ & 1.73 & 300.3 & 310.0 \\
\hline & 7 & $262.15 \pm 2.91$ & 1.19 & 258.4 & 266.4 \\
\hline & 14 & $236.15 \pm 4.43$ & 1.81 & 230.6 & 240.9 \\
\hline & 21 & $228.3 \pm 8.39$ & 3.42 & 218.1 & 238.8 \\
\hline & 28 & $196.5 \pm 4.42$ & 1.80 & 188.7 & 200.9 \\
\hline \multirow[t]{5}{*}{ Prestretched Ortho Organizer } & 1 & $227.7 \pm 1.86$ & 0.75 & 224.7 & 229.7 \\
\hline & 7 & $202.03 \pm 3.34$ & 1.36 & 196.2 & 205.5 \\
\hline & 14 & $170.51 \pm 3.27$ & 1.33 & 164.8 & 174.5 \\
\hline & 21 & $163.3 \pm 3.57$ & 1.45 & 158.8 & 168.8 \\
\hline & 28 & $148.2 \pm 1.80$ & 0.73 & 145.6 & 150.7 \\
\hline
\end{tabular}

SD: Standard deviation

Table 3: Mean force decay ( $\mathrm{cN}$ ) recorded in alcohol mixture $21.6 \%$ during different time durations for all elastomeric chains

\begin{tabular}{|c|c|c|c|c|c|}
\hline Alcohol mixture $21.6 \%$ & $\begin{array}{l}\text { Measurement } \\
\text { time points (day) }\end{array}$ & Mean $\pm S D$ & $\begin{array}{l}\text { Standard } \\
\text { error }\end{array}$ & Minimum & Maximum \\
\hline \multirow[t]{5}{*}{ Unstretched 3M Unitek } & 1 & $254.45 \pm 7.24$ & 2.95 & 249.4 & 268.3 \\
\hline & 7 & $243.75 \pm 3.98$ & 1.62 & 238.5 & 243.5 \\
\hline & 14 & $227.28 \pm 4.93$ & 2.01 & 220.1 & 232.4 \\
\hline & 21 & $203.5 \pm 2.16$ & 0.88 & 200.7 & 205.8 \\
\hline & 28 & $173.21 \pm 2.40$ & 0.98 & 170.8 & 176.7 \\
\hline \multirow[t]{5}{*}{ Prestretched 3M Unitek } & 1 & $213.51 \pm 1.67$ & 0.68 & 210.7 & 214.9 \\
\hline & 7 & $200.3 \pm 2.82$ & 1.15 & 197.4 & 205.6 \\
\hline & 14 & $190.75 \pm 1.55$ & 0.63 & 188.8 & 192.7 \\
\hline & 21 & $164.55 \pm 5.05$ & 2.06 & 158.2 & 170.5 \\
\hline & 28 & $142.88 \pm 5.81$ & 2.37 & 136.0 & 149.4 \\
\hline \multirow[t]{5}{*}{ Unstretched Ortho Plus } & 1 & $250.01 \pm 6.63$ & 2.70 & 238.6 & 256.7 \\
\hline & 7 & $240.65 \pm 2.97$ & 1.21 & 236.2 & 245.0 \\
\hline & 14 & $245.53 \pm 4.65$ & 1.90 & 239.4 & 250.3 \\
\hline & 21 & $191.38 \pm 2.52$ & 1.02 & 188.4 & 195.3 \\
\hline & 28 & $163.38 \pm 4.17$ & 1.70 & 158.6 & 168.5 \\
\hline \multirow[t]{5}{*}{ Prestretched Ortho Plus } & 1 & $213.68 \pm 2.06$ & 0.84 & 210.8 & 216.4 \\
\hline & 7 & $201.11 \pm 2.87$ & 1.17 & 197.5 & 205.6 \\
\hline & 14 & $191.41 \pm 2.44$ & 0.99 & 188.7 & 195.7 \\
\hline & 21 & $163.5 \pm 3.92$ & 1.603 & 158.8 & 169.6 \\
\hline & 28 & $141.1 \pm 2.40$ & 0.98 & 138.5 & 145.2 \\
\hline \multirow[t]{5}{*}{ Unstretched Ortho Organizer } & 1 & $243.64 \pm 5.72$ & 2.55 & 238.0 & 250.4 \\
\hline & 7 & $241.5 \pm 2.57$ & 1.05 & 238.6 & 245.7 \\
\hline & 14 & $214.45 \pm 4.07$ & 1.66 & 210.10 & 219.5 \\
\hline & 21 & $201.7 \pm 2.28$ & 0.93 & 198.9 & 205.6 \\
\hline & 28 & $181.38 \pm 2.47$ & 1.01 & 178.8 & 185.7 \\
\hline \multirow[t]{5}{*}{ Prestretched Ortho Organizer } & 1 & $213.41 \pm 1.07$ & 0.44 & 212.3 & 215.1 \\
\hline & 7 & $202.25 \pm 4.2$ & 1.71 & 197.5 & 208.2 \\
\hline & 14 & $191.20 \pm 3.29$ & 1.344 & 186.5 & 194.4 \\
\hline & 21 & $163.35 \pm 5.49$ & 2.24 & 155.1 & 169.5 \\
\hline & 28 & $142.7 \pm 3.24$ & 1.34 & 138.6 & 146.8 \\
\hline
\end{tabular}

SD: Standard deviation 
Table 4: Mean force decay (cN) recorded in alcohol mixture $8.38 \%$ during different time durations for all elastomeric chains

\begin{tabular}{|c|c|c|c|c|c|}
\hline Alcohol mixture $8.38 \%$ & $\begin{array}{l}\text { Measurement time } \\
\text { points (day) }\end{array}$ & Mean $\pm S D$ & $\begin{array}{l}\text { Standard } \\
\text { error }\end{array}$ & Minimum & Maximum \\
\hline \multirow[t]{5}{*}{ Unstretched 3M Unitek } & 1 & $305.23 \pm 4.51$ & 1.84 & 300.3 & 310.4 \\
\hline & 7 & $274.93 \pm 3.67$ & 1.49 & 270.4 & 279.3 \\
\hline & 14 & $255.28 \pm 1.76$ & 0.71 & 252.1 & 256.4 \\
\hline & 21 & $247.85 \pm 25.88$ & 10.56 & 235.4 & 300.6 \\
\hline & 28 & $211.00 \pm 3.92$ & 1.6 & 300.7 & 310.8 \\
\hline \multirow[t]{5}{*}{ Prestretched 3M Unitek } & 1 & $222.08 \pm 1.581$ & 0.64 & 220.7 & 224.4 \\
\hline & 7 & $204.51 \pm 5.33$ & 2.17 & 197.6 & 210.9 \\
\hline & 14 & $185.53 \pm 6.28$ & 2.56 & 188.8 & 192.7 \\
\hline & 21 & $173.16 \pm 3.98$ & 1.62 & 168.7 & 179.7 \\
\hline & 28 & $151.6 \pm 2.30$ & 0.94 & 148.7 & 154.4 \\
\hline \multirow[t]{5}{*}{ Unstretched Ortho Plus } & 1 & $276.48 \pm 1.67$ & 0.68 & 274.4 & 279.3 \\
\hline & 7 & $265.96 \pm 3.54$ & 1.44 & 260.7 & 269.5 \\
\hline & 14 & $233.48 \pm 2.11$ & 0.86 & 230.6 & 235.7 \\
\hline & 21 & $216.08 \pm 4.87$ & 1.99 & 210.10 & 220.7 \\
\hline & 28 & $196.9 \pm 2.67$ & 1.09 & 193.6 & 200.7 \\
\hline \multirow[t]{5}{*}{ Prestretched Ortho Plus } & 1 & $219.21 \pm 9.28$ & 3.78 & 200.6 & 225.7 \\
\hline & 7 & $202.85 \pm 2.97$ & 1.62 & 197.8 & 209.1 \\
\hline & 14 & $184.36 \pm 5.07$ & 2.07 & 176.7 & 189.0 \\
\hline & 21 & $190.7 \pm 4.72$ & 1.92 & 185.3 & 196.6 \\
\hline & 28 & $152.05 \pm 3.45$ & 1.40 & 148.0 & 156.7 \\
\hline \multirow[t]{5}{*}{ Unstretched Ortho Organizer } & 1 & $306.8 \pm 3.86$ & 1.73 & 300.3 & 310.0 \\
\hline & 7 & $275.1 \pm 3.22$ & 1.31 & 270.4 & 279.5 \\
\hline & 14 & $244.16 \pm 3.61$ & 1.47 & 240.5 & 249.9 \\
\hline & 21 & $233.10 \pm 2.82$ & 1.15 & 230.4 & 237.3 \\
\hline & 28 & $208.3 \pm 6.92$ & 2.82 & 200.7 & 216.3 \\
\hline \multirow[t]{5}{*}{ Prestretched Ortho Organizer } & 1 & $223.73 \pm 4.89$ & 1.99 & 220.0 & 233.2 \\
\hline & 7 & $200.95 \pm 2.84$ & 1.15 & 197.7 & 205.6 \\
\hline & 14 & $185.45 \pm 5.34$ & 2.18 & 178.10 & 190.9 \\
\hline & 21 & $172.48 \pm 5.31$ & 2.17 & 165.6 & 179.6 \\
\hline & 28 & $155.5 \pm 2.68$ & 1.09 & 152.8 & 159.2 \\
\hline
\end{tabular}

SD: Standard deviation

Table 5: Mean force decay $(\mathrm{cN})$ recorded artificial saliva during different time durations for all elastomeric chains

\begin{tabular}{|c|c|c|c|c|c|}
\hline Artificial saliva & $\begin{array}{l}\text { Measurement time } \\
\text { points (day) }\end{array}$ & Mean $\pm S D$ & $\begin{array}{l}\text { Standard } \\
\text { error }\end{array}$ & Minimum & Maximum \\
\hline \multirow[t]{5}{*}{ Unstretched 3M Unitek } & 1 & $284.9 \pm 4.001$ & 1.63 & 280.3 & 289.8 \\
\hline & 7 & $283.23 \pm 3.58$ & 1.46 & 280.1 & 289.0 \\
\hline & 14 & $283.98 \pm 2.72$ & 1.10 & 281.5 & 288.4 \\
\hline & 21 & $263.35 \pm 2.50$ & 1.02 & 260.4 & 266.4 \\
\hline & 28 & $272.91 \pm 2.77$ & 1.13 & 270.4 & 276.8 \\
\hline \multirow[t]{5}{*}{ Prestretched 3M Unitek } & 1 & $215.4 \pm 1.93$ & 0.78 & 212.3 & 217.4 \\
\hline & 7 & $210.83 \pm 3.46$ & 1.41 & 205.9 & 215.7 \\
\hline & 14 & $212.31 \pm 3.88$ & 1.58 & 206.5 & 215.7 \\
\hline & 21 & $193.15 \pm 5.93$ & 2.42 & 185.7 & 200.6 \\
\hline & 28 & $185.4 \pm 3.84$ & 1.56 & 179.6 & 189.7 \\
\hline \multirow[t]{5}{*}{ Unstretched Ortho Plus } & 1 & $277.5 \pm 2.87$ & 1.17 & 274.1 & 281.3 \\
\hline & 7 & $274.51 \pm 3.46$ & 1.41 & 270.5 & 279.5 \\
\hline & 14 & $274.08 \pm 3.47$ & 1.41 & 270.10 & 279.5 \\
\hline & 21 & $259.08 \pm 1.58$ & 0.64 & 256.8 & 260.9 \\
\hline & 28 & $258.75 \pm 2.79$ & 11.42 & 205.5 & $\begin{array}{l}289.1 \\
(\text { Cont'd...) }\end{array}$ \\
\hline
\end{tabular}




\begin{tabular}{|c|c|c|c|c|c|}
\hline Artificial saliva & $\begin{array}{l}\text { Measurement time } \\
\text { points (day) }\end{array}$ & Mean $\pm S D$ & $\begin{array}{l}\text { Standard } \\
\text { error }\end{array}$ & Minimum & Maximum \\
\hline \multirow[t]{5}{*}{ Prestretched Ortho Plus } & 1 & $214.98 \pm 2.15$ & 0.88 & 212.4 & 268.2 \\
\hline & 7 & $208.65 \pm 5.34$ & 2.18 & 212.7 & 215.6 \\
\hline & 14 & $214.08 \pm 3.28$ & 1.34 & 210.3 & 218.5 \\
\hline & 21 & $190.7 \pm 4.72$ & 1.92 & 185.3 & 196.6 \\
\hline & 28 & $185.53 \pm 2.61$ & 1.06 & 182.4 & 189.6 \\
\hline \multirow[t]{5}{*}{ Unstretched Ortho Organizer } & 1 & $284.58 \pm 4.63$ & 2.07 & 280.0 & 289.0 \\
\hline & 7 & $283.68 \pm 3.57$ & 1.45 & 280.6 & 289.1 \\
\hline & 14 & $283.35 \pm 2.79$ & 1.14 & 280.5 & 288.1 \\
\hline & 21 & $253.5 \pm 23.52$ & 9.6 & 205.8 & 266.9 \\
\hline & 28 & $273.06 \pm 2.62$ & 1.071 & 270.6 & 276.8 \\
\hline \multirow[t]{5}{*}{ Prestretched Ortho Organizer } & 1 & $216.13 \pm 1.89$ & 0.77 & 213.4 & 218.0 \\
\hline & 7 & $210.2 \pm 5.54$ & 2.26 & 202.4 & 216.0 \\
\hline & 14 & $213.16 \pm 4.72$ & 1.92 & 205.0 & 218.0 \\
\hline & 21 & $192.25 \pm 4.57$ & 1.86 & 185.5 & 197.0 \\
\hline & 28 & $184.9 \pm 3.56$ & 1.45 & 179.2 & 189.1 \\
\hline
\end{tabular}

SD: Standard deviation

Table 6: Results of the two-way ANOVA of elastomeric chain force decay for duration, solution, and interaction between variables

\begin{tabular}{|c|c|c|c|c|}
\hline & $\begin{array}{l}\text { Measurement } \\
\text { time points (day) }\end{array}$ & $\begin{array}{l}\text { Sum of } \\
\text { squares }\end{array}$ & F-statistics & $p$-value \\
\hline \multirow[t]{10}{*}{ Unstretched 3M Unitek } & 1 & 9183.17 & 98.9 & $<0.001$ \\
\hline & & 579.987 & & \\
\hline & 7 & 7766.35 & 119.2 & $<0.001$ \\
\hline & & 406.879 & & \\
\hline & 14 & 15430.1 & 186.5 & $<0.001$ \\
\hline & & 517.0 & & \\
\hline & 21 & 16214.7 & 155.6 & $<0.0001$ \\
\hline & & 651.1 & & \\
\hline & 28 & 89811.2 & 1616.34 & $<0.001$ \\
\hline & 347.27 & & & \\
\hline \multirow[t]{10}{*}{ Prestretched 3M Unitek } & 1 & 17074.9 & 1094.23 & $<0.0001$ \\
\hline & & 97.52 & & \\
\hline & 7 & 430.25 & 8.013 & $<0.0002$ \\
\hline & & 335.58 & & \\
\hline & 14 & 4092.59 & 77.23 & $<0.0001$ \\
\hline & & 331.17 & & \\
\hline & 21 & 4568.29 & 55.54 & $<0.0001$ \\
\hline & & 514.02 & & \\
\hline & 28 & 8026.07 & 103.06 & $<0.0001$ \\
\hline & & 486.72 & & \\
\hline \multirow[t]{11}{*}{ Unstretched Ortho Plus } & 1 & 5097.4 & 47.90 & $<0.0001$ \\
\hline & & 664.9 & & \\
\hline & 7 & 6250.2 & 166.74 & $<0.0001$ \\
\hline & & 234.5 & & \\
\hline & 14 & 16876.1 & 360224 & $<0.00001$ \\
\hline & & 292.80 & & \\
\hline & 21 & 18831.1 & 374.69 & $<0.0001$ \\
\hline & & 314.10 & & \\
\hline & 28 & 35797.6 & 51.42 & $<0.0001$ \\
\hline & & 4348.4 & & \\
\hline & & & & (Cont'd...) \\
\hline
\end{tabular}




\begin{tabular}{|c|c|c|c|c|}
\hline & $\begin{array}{l}\text { Measurement } \\
\text { time points (day) }\end{array}$ & $\begin{array}{l}\text { Sum of } \\
\text { squares }\end{array}$ & F-statistics & $p$-value \\
\hline \multirow[t]{10}{*}{ Prestretched Ortho Plus } & 1 & 847.37 & 10.14 & $<0.0001$ \\
\hline & & 521.92 & & \\
\hline & 7 & 513.75 & 9.0152 & $<0.0001$ \\
\hline & & 356.17 & & \\
\hline & 14 & 5227.54 & 104.67 & $<00001$ \\
\hline & & 312.143 & & \\
\hline & 21 & 6456.4 & 100.777 & $<00001$ \\
\hline & & 400.42 & & \\
\hline & 28 & 7607.57 & 213.6 & $<00001$ \\
\hline & & 222.5 & & \\
\hline \multirow[t]{10}{*}{ Unstretched Ortho Organizer } & 1 & 18184.8 & 197.50 & $<0.0001$ \\
\hline & & 575.45 & & \\
\hline & 7 & 8510.2 & 167.90 & $<0.0001$ \\
\hline & & 316.78 & & \\
\hline & 14 & 18717.4 & 309.9 & $<0.0001$ \\
\hline & & 377.4 & & \\
\hline & 21 & 10704.7 & 19.49 & $<0.001$ \\
\hline & & 3431.4 & & \\
\hline & 28 & 37050.1 & 445.43 & $<0.0001$ \\
\hline & & 515.22 & & \\
\hline \multirow[t]{10}{*}{ Prestretched Ortho Organizer } & 1 & 1321.73 & 28.68 & $<0.0001$ \\
\hline & & 287.95 & & \\
\hline & 7 & 575.38 & 9.89 & $<0.0001$ \\
\hline & & 363.30 & & \\
\hline & 14 & 7694.67 & 113.27 & $<0.0001$ \\
\hline & & 424.54 & & \\
\hline & 21 & 4857.44 & 58.68 & $<0.0001$ \\
\hline & & 517.29 & & \\
\hline & 28 & 7041.79 & 201.68 & $<0.0001$ \\
\hline & & 217.89 & & \\
\hline
\end{tabular}

The difference in mean force decay among the different time intervals was found to be statistically significant $(p<0.001)$. Among the different solutions, higher mean force was recorded in $21.6 \%$ alcohol mixture $>$ Listerine $8.38 \%>$ Wokadine $>$ alcohol mixture $8.38 \%>$ artificial saliva. The difference in mean force decay among the different solutions was found to be statistically significant $(p<0.001)$. The interaction of duration and solution on mean force decay was found to be statistically significant $(p<0.001)$.

\section{DISCUSSION}

Elastomeric chains are used as the retractive force to move teeth into extraction sites, closing diastemas, generalized space closure, and correction of rotated teeth. The chains serve as ligatures to tie the archwire to the bracket.

Since the 1970s, several studies have been published on the decay of elastomers in the oral cavity during orthodontic treatment. ${ }^{5,6}$ The results of various studies on orthodontic elastics have shown wide range of force decay (24-85\%) after 28 days. Some of the reasons behind these results may include difference in the media in which the samples were tested and quality of the elastomeric chains used.

Years ago, Baty et $\mathrm{al}^{7}$ evaluated the behavior of elastics over 3 weeks. However, a period of 4 weeks was selected for the study because it coincides with the time interval between orthodontic consultations; the same period was observed by Motta et al. ${ }^{8}$

The mean percentage of force loss after 24 hours was 41.68 to $55.18 \%$ for the tested groups, which is comparable to that reported in the published studies for elastomeric chains. ${ }^{1,2,9,10}$ Alcohol $21.6 \%$ had the highest percentage of force decay, i.e., $55.18 \%$ for short module after 24 hours due to hydrolysis caused by alcohol. Wokadine showed the lowest percentage of force decay of $41.68 \%$.

Larrabee et $\mathrm{al}^{4}$ had found a force decay of $53.0 \%$ in Listerine mouth rinse after 24 hours. A rapid initial force loss within 24 hours could increase patient comfort and initial tissue response without affecting tooth-moving ability. Highest mean residual force was recorded in 
artificial saliva followed by Wokadine, alcohol mixture $8.38 \%$, Listerine, and alcohol mixture $21.6 \%$ respectively. The difference in mean force decay among the different solutions was found to be statistically significant $(p<0.001)$.

De Genova et $\mathrm{al}^{1}$ found that elastomeric chains producing higher initial forces displayed less force decay compared with the chains producing lesser initial force. However, the results obtained in the present study do not support these findings, possibly due to the methodological differences between the two studies. The forces exerted after 28 days in this study did not drop to zero as found in a clinical study by Samuels et al. ${ }^{11}$

In the Samuels et al study, ${ }^{11}$ forces were tested between 5 and 8 weeks, whereas in this study, forces were tested at 28 days after exposure to a simulated oral environment.

Among the durations, higher mean force was recorded initially followed by 24 hours, 7 days, 14 days, 21 days, and 28 days respectively. The difference in mean force decay among the different time intervals was found to be statistically significant $(p<0.001)$. Among Listerine and Wokadine mouth rinses, higher mean residual force was recorded in Wokadine, which has high antimicrobial activity. The interaction (joint effect) of duration and solution on mean force decay was also found to be statistically significant $(\mathrm{p}<0.001)$.

The results of this study demonstrate that active components in mouth rinses cause lesser amount of force decay in elastomeric chains exposed to $21.6 \%$ alcohol, $8.38 \%$ alcohol than when it is exposed to commercial mouth rinses as a whole. The results also confirm previous studies, ${ }^{1,12-14}$ showing force decay over time, which is high initially and then reaches a plateau.

Kim et $\mathrm{a}^{15}$ found effects of prestretching on timedependent force decay of synthetic elastomeric chains. Elastomeric chains displayed less force decay $(8.0 \%)$ after 24 hours; in the present study, after 24 hours in Listerine with prestretched 3M Unitek $40.58 \%$ force loss occurred and unstretched elastomeric chains show more force decay than elastomeric prestretched chains, but the results obtained in the present study do not support these findings, possibly due to the methodological differences between the two studies.

Limitations of this study are that since this study was done under static conditions and in vitro, it performed short of an exact picture of the degradation in vivo. Further studies need to be conducted in clinical situation where varied oral environment due to the different dietary habits, microbial activity, and stretching is present.

\section{CONCLUSION}

Alcohol-containing mouth rinses cause an increase in force decay of elastomeric chain over time. At the end of 28 days, Listerine $(69.25 \%)$ showed more force decay of elastomeric chain and Wokadine $(64.54 \%)$ showed less force decay of elastomeric chain. Among all three elastomeric chains at the end of 28 days, more force decay occurred in unstretched Ortho Plus and less in prestretched 3M Unitek. Unstretched elastomeric chains show more force decay than elastomeric prestretched chains.

\section{REFERENCES}

1. De Genova DC, McInnes-Ledoux P, Weinberg R, Shaye R. Force degradation of orthodontic elastomeric chains - a product comparison study. Am J Orthod 1985 May;87(5):377-384.

2. Ferriter JP, Meyers CE Jr, Lorton L. The effect of hydrogen ion concentration on the force-degradation rate of orthodontic polyurethane chain elastics. Am J Orthod Dentofacial Orthop 1990 Nov;98(5):404-410.

3. Taloumis LJ, Smith TM, Hondrum SO, Lorton L. Force decay and deformation of orthodontic elastomeric ligatures. Am J Orthod Dentofacial Orthop 1997 Jan;111(1):1-11.

4. Larrabee TM, Liu SS, Torres-Gorena A, Soto-Rojas A, Eckert GJ, Stewart KT. The effects of varying alcohol concentrations commonly found in mouth rinses on the force decay of elastomeric chain. Angle Orthod 2012 Sep;82(5):894-889.

5. Balhoff DA, Shuldberg M, Hagan JL, Ballard RW, Armbruster PC. Force decay of elastomeric chains - a mechanical design and product comparison study. J Orthod 2011 Mar;38(1):40-47.

6. Lu TC, Wang WN, Tarng TH, Chen JW. Force decay of elastomeric chain - a serial study. Part II. Am J Orthod Dentofacial Orthop 1993 Oct;104(4):373-377.

7. Baty DL, Storie DJ, von Fraunhofer JA. Synthetic elastomeric chains: a literature review. Am J Orthod Dentofacial Orthop 1994 Jun;105(6):536-542.

8. Motta AF, Cury-Saramago AA, Nojima LI. In vitro evaluation of force delivered by elastic chains. Dent Press J Orthod 2011 Nov-Dec;16(6):36.e1-36.e8.

9. Brantley WA, Salander S, Myers CL, Winders RV. Effects of prestretching on force degradation characteristics of plastic modules. Angle Orthod 1979 Jan;49(1):37-43.

10. von Fraunhofer JA, Coffelt MT, Orbells GM. The effects of artificial saliva and topical fluoride treatments on the degradation of the elastic properties of orthodontic chains. Angle Orthod 1992 Winter;62(4):265-274.

11. Samuels RH, Rudge SJ, Mair LH. A comparison of the rate of space closure using a nickel-titanium spring and an elastic module: a clinical study. Am J Orthod Dentofacial Orthop 1993 May;103(5):464-467.

12. Hain M, Dhopatkar A, Rock P. The effect of ligation method on friction in sliding mechanics. Am J Orthod Dentofacial Orthop 2003 Apr;123(4):416-422.

13. Kersey ML, Glover KE, Heo G, Raboud D, Major PW. An in vitro comparison of 4 brands of nonlatex orthodontic elastics. Am J Orthod Dentofacial Orthop 2003 Apr;123(4):401-407.

14. Elidias T, Silikas N, Watts DC. In vitro degradation of polyurethane orthodontic elastomeric modules. J Oral Rehabil 2005 Jan;32(1):72-77.

15. Kim $\mathrm{KH}$, Chung $\mathrm{CH}$, Choy $\mathrm{K}$, Lee JS, Vanarsdall RL. Effects of prestretching on force degradation of synthetic elastomeric chains. Am J Orthod Dentofacial Orthop 2005 Oct;128(4):477-482. 